\title{
Characterizing AGB stars in Wide-field Infrared Survey Explorer (WISE) bands ${ }^{\star}$
}

\author{
Jianhui Lian ${ }^{1,2}$, Qingfeng Zhu ${ }^{1,2}$, Xu Kong ${ }^{1,2}$, and Jinhua $\mathrm{He}^{3}$ \\ ${ }^{1}$ Center for Astrophysics, University of Science and Technology of China, 230026 Hefei, PR China \\ e-mail: 1jhhw@mail.ustc.edu.cn; [zhuqf; xkong] @ustc.edu.cn \\ 2 Key Laboratory for Research in Galaxies and Cosmology, Chinese Academy of Sciences, 230026 Hefei, PR China \\ 3 Key Laboratory for the Structure and Evolution of Celestial Objects, Yunnan Observatories, CAS, 650011 Kunming, PR China
}

Received 8 October 2013 / Accepted 20 February 2014

\begin{abstract}
Aims. Since asymptotic giant branch (AGB) stars are bright and extended infrared objects, most Galactic AGB stars saturate the Wide-field Infrared Survey Explorer (WISE) detectors and therefore the WISE magnitudes that are restored by applying point-spreadfunction fitting need to be verified. Statistical properties of circumstellar envelopes around AGB stars are discussed on the basis of a WISE AGB catalog verified in this way.

Methods. We cross-matched an AGB star sample with the WISE All-Sky Source Catalog and the Two Mircon All Sky Survey catalog. Infrared Space Observatory (ISO) spectra of a subsample of WISE AGB stars were also exploited. The dust radiation transfer code DUSTY was used to help predict the magnitudes in the $W 1$ and $W 2$ bands, the two WISE bands most affected by saturation, for calibration purpose, and to provide physical parameters of the AGB sample stars for analysis.

Results. DUSTY is verified against the ISO spectra to be a good tool to reproduce the spectral energy distributions of these AGB stars. Systematic magnitude-dependent offsets have been identified in WISE $W 1$ and $W 2$ magnitudes of the saturated AGB stars, and empirical calibration formulas are obtained for them on the basis of 1877 (W1) and 1558 (W2) AGB stars that are successfully fit with DUSTY. According to the calibration formulas, the corrections for $W 1$ at 5 mag and $W 2$ at 4 mag are -0.383 and 0.217 mag, respectively. In total, we calibrated the $W 1 / W 2$ magnitudes of 2390/2021 AGB stars. The model parameters from the DUSTY and the calibrated WISE $W 1$ and $W 2$ magnitudes are used to discuss the behavior of the WISE color-color diagrams of AGB stars. The model parameters also reveal that O-rich AGB stars with opaque circumstellar envelopes are much rarer than opaque C-rich AGB stars toward the anti-Galactic center direction, which we attribute to the metallicity gradient of our Galaxy.
\end{abstract}

Key words. stars: AGB and post-AGB - stars: carbon - stars: evolution - infrared: stars - radiative transfer

\section{1. introduction}

The asymptotic giant branch (AGB) phase is the final stellar evolutionary stage of intermediate-mass $\left(1-8 M_{\odot}\right)$ stars driven by nuclear burning. Stars in this stage have low surface effective temperatures (below $3000 \mathrm{~K}$ ) and experience intense mass loss (from $10^{-7}$ to $10^{-4} M_{\odot} \mathrm{yr}^{-1}$ ) (Habing 1996). Heavy elements in the mass outflow from a central star will condense to form dust when the gas temperature drops to the sublimation temperature of the dust grains. Dusty circumstellar envelopes will form at the distance of several stellar radii. Dust grains in the envelopes absorb stellar radiation and re-emit in the infrared. Thus, AGB stars are important infrared sources. The mass-loss process plays an important role in the evolution of AGB stars because it affects the lifetime of the AGB phase and the core-mass of the subsequent post-AGB stars. Statistics of a large sample of AGB stars would help to constrain the evolution of dust envelope. Due to the relative over-abundance between carbon and oxygen, there are two main types of AGB stars: (1) the O-rich with $\mathrm{C} / \mathrm{O}<1$ and mainly silicate-type grains in the outflow; and (2) C-rich with $\mathrm{C} / \mathrm{O}>1$ and mainly carbonaceous grains in the envelopes.

\footnotetext{
* The synthetic photometry and input parameters for the model grid are only available at the CDS via anonymous ftp to

cdsarc.u-strasbg.fr (130.79.128.5) or via

http://cdsarc.u-strasbg.fr/viz-bin/qcat?]/A+A/564/A84
}

The different dust compositions of these two types of AGB stars result in different infrared spectral features, which can be used to distinguish the two groups of the stellar objects.

The first-generation infrared space-telescope, the Infrared Astronomical Satellite (IRAS; Neugebauer et al. 1984), mapped the sky in two mid-infrared and two far-infrared bands (12, $25 \mu \mathrm{m}$ and $60,100 \mu \mathrm{m}$, respectively) in the 1980s. It discovered many mass-losing AGB stars in the Milky Way and the Magellanic Clouds. Several dozen AGB stars were observed spectroscopically by the Infrared Space Observatory (ISO; Kessler et al. 1996) with the Short-Wave Spectrometer (SWS) on board, which works in the range of $2.4 \mu \mathrm{m}$ to $45 \mu \mathrm{m}$. These infrared spectra provide tremendous information about dusty circumstellar envelopes around the AGB stars, but they are limited by the sample size. The Galactic Plane Survey of the Spitzer Space Telescope (Werner et al. 2004) provides highresolution and sensitive infrared images of Galactic plane at the 3.6, 4.5, 5.8, 8.0, 24, and $70 \mu \mathrm{m}$ bands. However, it cannot adequately separate the two types of AGB stars in mid-infrared color-color diagrams without filters between $8 \mu \mathrm{m}$ and $24 \mu \mathrm{m}$. Launched on 2009 December 14, the Wide-field Infrared Survey Explorer (WISE) completed an entire sky survey in the 3.4, 4.6, 12 and $22 \mu \mathrm{m}$ bands (hereafter named $W 1, W 2, W 3$, and $W 4$, respectively) in 2010. The FWHMs of the point spread functions (PSF) are 6"' 1, 6!' 4, 6"' 5 , and 12'” 0 for the four WISE bands, 
and the sensitivities $(5 \sigma)$ of the point sources are $0.08,0.11,1$, and 6 mJy (Wright et al. 2010). The Two Micron All Sky Survey (2MASS; Skrutskie et al. 2006) also mapped the entire sky in three near-infrared, $J(1.25 \mu \mathrm{m}), H(1.65 \mu \mathrm{m})$ and $K_{\mathrm{s}}(2.17 \mu \mathrm{m})$ bands. They are an important supplement to the mid-infrared WISE data to complete the infrared spectral energy distribution (SED) of AGB stars.

Recently, galaxy-wide AGB populations have been identified and studied in nearby galaxies using ground- and space-based near- and mid-infrared data (Riebel et al. 2012; Boyer et al. 2012 in the Magellanic Clouds and Javadi et al. 2013 in M33). While similar research in our galaxy is hindered by foreground extinction, there are some studies on large datasets of Galactic AGB stars, such as the work of Jura \& Kleinmann (1989) in the solar neighborhood. Suh \& Kwon $(2009,2011)$ composed a large verified AGB catalog in our galaxy from the literature and investigated infrared colors of AGB stars and their distribution in color-color diagrams. To study an AGB star population, a more time-saving way is constructing a set of model grids to simulate the infrared SED of AGB stars and acquire their physical parameters, such as mass-loss rate and optical depth. Many model grids have been developed to study the evolved-star populations in the local group galaxies (Groenewegen 2006; Sargent et al. 2011; Srinivasan et al. 2011). All of the models are publicly available. However, the model set of Groenewegen (2006) does not have WISE synthetic photometry. The Magellanic Clouds have a lower metallicity than our Galaxy, and Sargent et al. (2011) and Srinivasan et al. (2011) chose a stellar photosphere model with subsolar metallicity as input of a radiative transfer model, which is not the case in our galaxy. Therefore, we used DUSTY (Ivezic \& Elitzur 1997) to generate a new grid of radiative transfer models to simulate the infrared SEDs of Galactic AGB stars.

As AGB stars typically are bright IR objects, most of our AGB samples are brighter than the WISE detector saturation limits and only photometry of unsaturated pixels are available, therefore their WISE magnitudes need to be verified. The main goal of this paper is to study the properties of AGB stars in WISE bands and recalibrate the WISE photometry for sources saturated in WISE bands. We describe our AGB sample, DUSTY algorithm and calibration method in Sect. 2. A possible calibrating solution is presented in Sect. 3.1. We present our results about the distinct location of the two types of AGB stars in a color-color diagram in Sect. 3.2, the model parameter effects on WISE colors in Sect. 3.3, and the Galactic longitude distribution in Sect. 3.4. Our summary is presented in Sect. 4. All the magnitudes mentioned in this paper are Vega magnitudes.

\section{Sample and method}

\subsection{AGB sample}

Suh \& Kwon (2009) composed a catalog of Galactic AGB stars based on reports of the IRAS Low Resolution Spectrograph (LRS; $\lambda=8-22 \mu \mathrm{m}$ ), ISO Short-Wave Spectrometer (SWS), the Near Infrared Spectrometer (NIRS; $1.4-4.0 \mu \mathrm{m}$; Murakami et al. 1994), radio OH and SiO survey (Lewis et al. 1990), the Midcourse Space Experiment (MSX; Egan et al. 2003) and the 2MASS data. Suh \& Kwon (2011) (hereafter Suh11) updated the catalog with $\mathrm{SiO}$ maser sources for O-rich AGB stars and additional sources for C-rich AGB stars. The updated catalog contains 3003 O-rich and 1168 C-rich AGB stars with their IRAS colors and has the largest confirmed AGB collection so far. This catalog provides an important entry for the future study of AGB stars.
To study the properties of AGB stars in infrared bands, we cross-correlated the AGB catalog with the WISE All Sky Catalog. Because IRAS data have a positional uncertainty ellipse of $45^{\prime \prime} \times 9^{\prime \prime}$, which is much bigger than the position uncertainty in WISE photometry $\left.(\sim 0)^{\prime} 20\right)$, we used coordinates and magnitudes of sources during the cross-correlation to improve the precision. We first used $45^{\prime \prime}$ as the searching radius to find matching candidates. In the cases of multiple matches, we selected the WISE source with the highest $12 \mu \mathrm{m}$ flux. AGB stars are among the brightest sources in the sky at midinfrared wavelengths. The detection limit of IRAS is higher than that of WISE. All $12 \mu \mathrm{m}$ IRAS sources needed also to be detected by WISE. However, high flux saturates the WISE detectors and may render them unreliable. A selection criterion of W3 fainter than -2 mag was applied to exclude other 70 O-rich and 33 C-rich AGB stars from the sample. This criterion is based on our analysis of WISE magnitudes and ISO SWS spectra of AGB stars, which is discussed in Sect. 2.3. The 2MASS observations in $J, H$ and $K_{\mathrm{s}}$ bands are included in the WISE catalog. For each WISE source, the associated 2MASS observations were selected with the nearest sources in the 2MASS catalog within $3^{\prime \prime}$. Some of these AGB stars (730 O-rich and 158 C-rich) do not have a 2 MASS counterpart, which is perhaps due to heavy circumstellar extinction. This tentative interpretation is supported by the fact that their $W 3-W 4$ colors (with medians of 1.874 mag for O-rich and $0.993 \mathrm{mag}$ for C-rich objects), which correspond to an optical depth $\sim 40$ at $0.55 \mu \mathrm{m}$ (according to our discussion in Sect. 3.2), are much redder than that of the whole AGB sample $(W 3-W 4=1.314 \mathrm{mag}$ for O-rich and $0.454 \mathrm{mag}$ for $\mathrm{C}$-rich objects). Finally, 2203 (73.6\%) O-rich and 958 (83.6\%) C-rich AGB stars with 2MASS observations remained. They are our WISE AGB sample. We did not apply interstellar extinction correction to our data because there are no distance estimates. The extinction caused by the interstellar material (ISM) can be included in the extinction of the dust envelope around AGB stars. We expect that the optical depth is overestimated by a factor depending on the distance to the Sun and specific sightlines. The good agreement between DUSTY models and ISO data suggests that our results from estimating the saturated WISE $W 1$ and $W 2$ photometry are not affected by interstellar extinction significantly.

To calibrate the WISE photometry, we cross-matched the Suh11 catalog with sources observed by ISO SWS (spectrum data reduced by Sloan et al. 2003). To obtain a reliable spectral sample, we excluded several O-rich AGB star spectra that deviate significantly from the typical SEDs of AGB stars and lack $10 \mu \mathrm{m}$ silicate feature. Spectra with a low signal-to-noise ratio were also excluded. Finally, we obtained 67 O-rich and 44 $\mathrm{C}$-rich AGB stars with reliable ISO mid-infrared spectra. Among them, 66 (49) O-rich and 44 (37) C-rich AGB stars have associated WISE (and 2MASS) observations.

\subsection{Theoretical modeling}

DUSTY is a radiative transfer code developed by Ivezic \& Elitzur (1997) and can be used to model the dusty circumstellar envelope around AGB stars. Many authors have used this code to simulate the infrared emission of AGB stars (e.g., Riechers et al. 2004; Matsuura et al. 2006; Groenewegen 2012). Taking the advantage of scaling, Ivezic \& Elitzur (1997) minimized the number of input parameters by assuming spherical symmetry. The parameters that have to be specified in models are (1) the input radiation field, which can be described by the effective temperature $T_{\text {eff }}$ of the cental star; (2) the optical properties of dust 
grains with a specified chemical composition; (3) the grain-size distribution $n(a)$; (4) the dust temperature at the inner boundary of the circumstellar envelope $T_{\text {in }}$; (5) the relative thickness (radius at the outer boundary over radius at the inner boundary, $r_{\text {out }} / r_{\text {in }}$ ) of the envelope; (6) envelope density distribution assuming a power law of $\rho(r) \propto r^{-\alpha}$ in this work; (7) the overall optical depth at a reference wavelength $\tau_{\lambda}$, and we assumed a reference wavelength at $0.55 \mu \mathrm{m}$.

We used DUSTY to generate model templates to simulate the infrared SEDs of AGB stars in our Galaxy. For the radiative field, we took the COMARCS hydrostatic models (Aringer et al. 2009) of AGB star photospheres to represent the C-rich AGB stars and PHOENIX models (Kučinskas et al. 2005) for O-rich AGB stars. Both models assume metallicity $Z=Z_{\odot}$. As noted in Aringer et al. (2009), most C-rich AGB stars have temperatures lower than $3500 \mathrm{~K}$; the effective temperature of C-rich stellar models in this work ranges from $2500 \mathrm{~K}$ to $3500 \mathrm{~K}$ with increments of $500 \mathrm{~K}$. For O-rich AGB stars, we used PHOENIX models with stellar effective temperatures between $2100 \mathrm{~K}$ and $4500 \mathrm{~K}$ in increments of $400 \mathrm{~K}$. The effective temperature increments are larger than in the model grids of Sargent et al. (2011) and Srinivasan et al. (2011), which are 200 and $100 \mathrm{~K}$, respectively, to save calculation time. We explored the effective temperature with increments as small as $100 \mathrm{~K}$ and did not find a noticeable increase in the number of AGB stars with good fits. We set the stellar mass for O-rich AGB stars to be $1 M_{\odot}$, which is the only available choice in the PHOENIX model and a better choice than a blackbody SED. As shown in Aringer et al. (2009) and Kučinskas et al. (2005), the surface gravities $\left(\log \left(\mathrm{g} /\left(\mathrm{cm} \mathrm{s}^{-2}\right)\right)\right)$ and $\mathrm{C} / \mathrm{O}$ ratio have a minor effect on nearinfrared colors, therefore we only used COMARCS models with $\log (g)=-0.4$ (when the temperature was higher than $3200 \mathrm{~K}$, only $\log (g)=-0.2$ is available) and $\mathrm{C} / \mathrm{O}=1.1$, and PHOENIX models with $\log (g)=-0.5$ and $\mathrm{C} / \mathrm{O}=1.1$.

Suh11 also used DUSTY to simulate the infrared colors of AGB stars. They assumed that each type of AGB circumstellar envelope contains only two types of dust grains. The circumstellar envelopes of O-rich AGB stars only contain silicate and porous corundum $\mathrm{Al}_{2} \mathrm{O}_{3}$. The circumstellar envelopes of C-rich AGB stars contain amorphous carbon (AmC) and SiC. A composition ratio parameter $X$ was defined to specify the number ratio of the two types of dust particles. In O-rich AGB stars, $X=$ silicate $/\left(\right.$ silicate $\left.+\mathrm{Al}_{2} \mathrm{O}_{3}\right)$; in C-rich AGB stars, $X=\mathrm{AmC} /(\mathrm{AmC}+\mathrm{SiC})$. They found that this assumption can well describe the properties of the envelopes of AGB stars in general. In many previous studies of radiative transfer models of AGB stars, the dust composition ratio $X$ is fixed. For example, Sargent et al. (2011) used $100 \%$ silicate for O-rich AGB stars and Srinivasan et al. (2011) used a mixture consisting of $90 \% \mathrm{AmC}$ and $10 \% \mathrm{SiC}$ for C-rich AGB stars. Groenewegen (2006) introduced three dust composition ratios $(X=100 \%$, $40 \%$ and $0 \%)$ for O-rich and two ratios ( $X=100 \%$ and $85 \%)$ for $\mathrm{C}$-rich envelopes. In this work, to save time and for completeness, we fixed the dust ratio to $100 \%$ silicate around O-rich AGB stars and adopted five dust composition ratios of AmC and SiC ranging from $100 \%$ to $60 \%$ for C-rich stars. The optical properties of silicate grains were taken from Ossenkopf et al. (1992) and the porous corundum $\mathrm{Al}_{2} \mathrm{O}_{3}$ from Begemann et al. (1997). While the optical properties of porous corundum from Begemann et al. (1997) only cover a wavelength range redward of $7.8 \mu \mathrm{m}$, the refraction indices are assumed to be constant at shorter wavelengths, with a value equal to the corresponding end point. We can see from Fig. 2 of Begemann et al. (1997) that the refractive index from Koike et al. (1995) and Chu et al. (1988)
Table 1. Parameter range for model templates.

\begin{tabular}{lcccc}
\hline \hline & O-rich range & Increment & C-rich range & Increment \\
\hline$T_{\text {eff }}(\mathrm{K})$ & $2100-4500$ & 400 & $2500-3500$ & 500 \\
$X$ & $100 \%$ & $\ldots$ & $100 \%-60 \%$ & $20 \%$ \\
$T_{\text {in }}(\mathrm{K})$ & $600-1400$ & 200 & $600-1800$ & 200 \\
$\log \left(\tau_{0.55}\right)$ & $-2-2$ & $0.3^{a}$ & $-2-2$ & $0.3^{a}$ \\
\hline
\end{tabular}

Notes. ${ }^{(a)}$ The increments for $\log \left(\tau_{0.55}\right)$ in intervals of $[-2,0.7]$, $[0.8,1.4]$, and $[1.45,2]$ are $0.3,0.1$, and 0.05 , respectively.

at wavelengths shorter than $7.8 \mu \mathrm{m}$ is roughly constant. The optical properties of AmC were taken from Suh (2000) and SiC from Pegourie (1988). The grain-size distribution was modeled as $n(a) \propto a^{-q}$ for $a_{\min }<a<a_{\max }$ (MRN distribution, Mathis et al. 1977) with a power-law index $q=3.5, a_{\min }=0.005 \mu \mathrm{m}$ and $a_{\max }=0.25 \mu \mathrm{m}$.

In DUSTY models, $T_{\text {in }}$ is specified directly instead of the inner radius. We calculated models with $T_{\text {in }}$ ranging from 600-1800 K for C-rich and 600-1400 K for O-rich AGB stars with increments of $200 \mathrm{~K}$. Dust grains cannot form at temperatures higher than the sublimation temperature, which is approximately $1400 \mathrm{~K}(1800 \mathrm{~K})$ for silicate (graphite grains) (Posch et al. 2007; Speck et al. 2009). The ranges of $T_{\text {in }}$ are roughly consistent with that of models in Sargent et al. (2011) and Srinivasan et al. (2011). We assumed that the power of the density profile $\alpha=2$ for the circumstellar envelopes, which is typical for steady circumstellar winds. When $\alpha=2$, we find that the variance of the relative thickness of the shell from $10^{2}$ to $10^{5}$ does not have a significant impact on the mid-infrared colors at WISE wavelengths. Thus, the relative thickness was fixed at $4 \times 10^{3}$. The optical depth $\tau$ at $0.55 \mu \mathrm{m}$ ranges from $10^{-2}$ to $10^{2}$ and is sampled in logarithmic space, with a finer stepsize at the highest optical depths to ensure an even distribution of models in color-color space. Finally, we calculated 3 (7) values of $T_{\text {eff }}, 5$ (1) values of $X, 7$ (5) values of $T_{\text {in }}$, and 29 (29) values of $\tau_{0.55}$ for C-rich (O-rich) AGB stars. This gives a total of 3045 and 1015 models for C-rich and O-rich AGB stars, respectively. The ranges of model parameters are listed in Table 1.

Given each set of parameters of $T_{\text {eff }}, \mathrm{X}, T_{\text {in }}, r_{\text {out }} / r_{\text {in }}, \alpha, \tau_{\lambda}$, DUSTY computes the emerging SED (from $0.1 \mu \mathrm{m}$ to $3600 \mu \mathrm{m}$ ) from the envelope. The SED consists of three components: attenuated stellar radiation, scattered radiation, and dust emission. The emerging SED can be convolved with WISE photoncounting relative system response (RSR) curves, $\lambda R(\lambda)$ (Jarrett et al. 2011), to simulate the WISE photometry:

$W_{n}=-2.5 \log \left(\frac{\int F_{\lambda} \lambda R_{\lambda} \mathrm{d} \lambda}{F_{\lambda n} b_{n}}\right) \quad n=1,2,3,4$.

Here $b_{n}$ is the width (in units of $\mu \mathrm{m}$ ) of each WISE band. $F_{\lambda n}$ are the fluxes at the magnitude zero point in the four bands. Since WISE saturates on Vega, the magnitude zero points are based on fluxes of fainter stars calibrated to the Vega system. We applied 2MASS relative spectral response curves derived by Cohen et al. (2003) to obtain the simulated 2MASS magnitudes. This procedure was used to simulate the WISE and 2MASS magnitudes for each model template.

\subsection{WISE photometry and calibration method}

We applied the WISE RSRs to the ISO spectra to simulate WISE photometry for these ISO objects. Figure 1 compares 

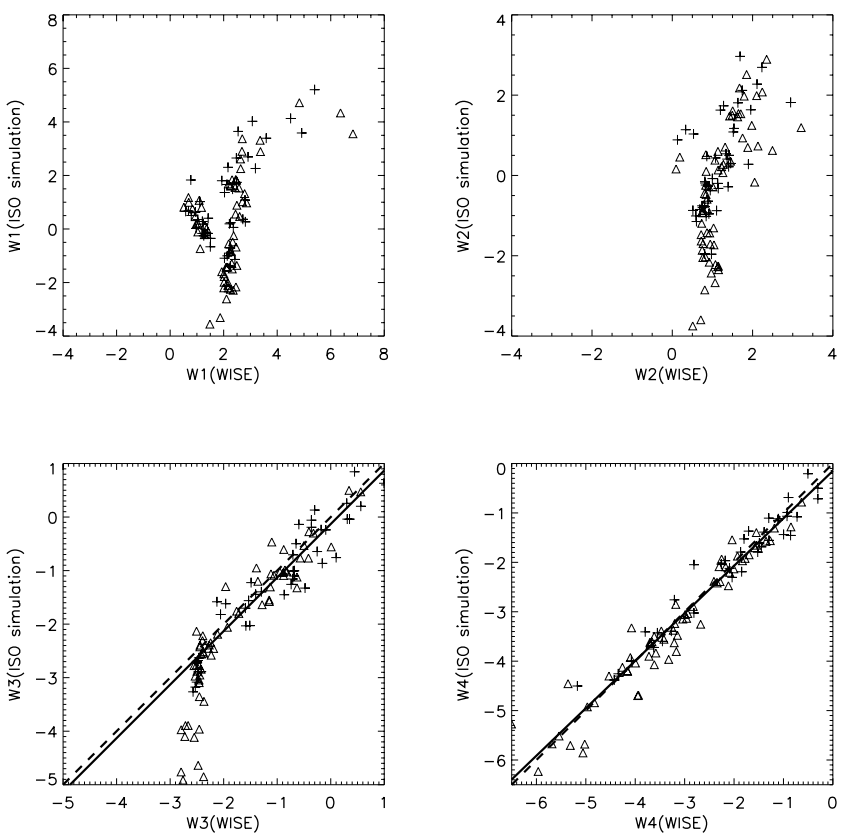

Fig. 1. Comparison between the WISE magnitudes and the ISO spectral observations of AGB stars in WISE $W 1-W 4$ bands. Pluses are O-rich and triangles are C-rich AGB stars. Dashed lines indicate equality between the $x$ - and $y$-axis values, and solid lines are the best-fit linear relations where applicable.

the observed WISE magnitudes with those simulated from the ISO spectra. In the figure, the 66 O-rich stars are designated as plus signs and the $44 \mathrm{C}$-rich AGB stars as triangles. Dashed lines indicate equality between the $x$ - and $y$-axis values. Solid lines in $W 3$ and $W 4$ panels are the best-fit relations given by

$$
\begin{aligned}
W 3(\mathrm{ISO})= & -0.13( \pm 0.26) \\
& +1.00( \pm 0.00) \times W 3(\mathrm{WISE}) \\
W 4(\mathrm{ISO})= & -0.15( \pm 0.09) \\
& +0.95( \pm 0.09) \times W 4(\mathrm{WISE}) .
\end{aligned}
$$

We fit the line equation for $W 3$ with a fixed unity slope to safely extrapolate the relation to fainter magnitude. The correlation between ISO and WISE is quite good in the $W 3$ and $W 4$ bands, except that a turning point exists in the $W 3$ band. The correlation only holds for objects fainter than -2 mag in $W 3$. That is the reason why we excluded sources with $W 3$ brighter than -2 when we assembled our WISE AGB sample in Sect. 2.1. As ISO and WISE observations were taken at different epochs and AGB stars are variable objects even in infrared bands, part of the spread in the $W 3$ and $W 4$ equation is due to the variability of AGB stars. We also compared WISE $W 3$ and $W 4$ bands with IRAS $12 \mu \mathrm{m}$ and $25 \mu \mathrm{m}$ data and found they agree well. However, no obvious correlation is observed between WISE data and ISO observations in the $W 1$ and $W 2$ bands.

It is mentioned in the WISE Explanatory Supplement documentation of the All-Sky Data Release that bright sources will saturate WISE detectors ${ }^{1}$. The saturation limits are 8.1, 6.7, 3.8, and $-0.4 \mathrm{mag}$ for the $W 1-W 4$ bands, respectively. For saturated objects, WISE fits the PSF to the unsaturated pixels on the images to recover the saturatured pixels and yield photometry for these objects (we call this PSF-fit photometry). The bright

\footnotetext{
1 WISE Explanatory Supplement documentation for All-Sky Data Release http://wise2.ipac.caltech.edu/docs/release/ allsky/expsup/
}
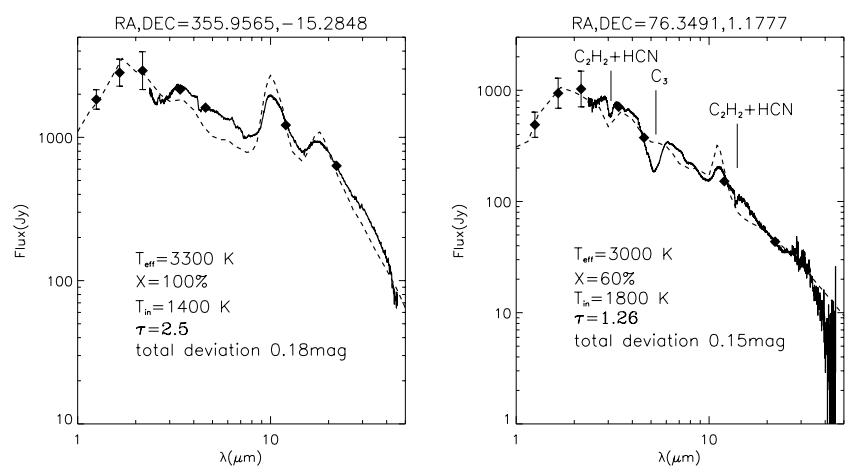

Fig. 2. Comparison between photometric/spectroscopic observations and DUSTY best-fit models for AGB stars. The left panel is for a selected O-rich star and the right panel for a C-rich star. The solid lines are ISO SWS spectra in the range between $2.5 \mu \mathrm{m}$ and $45 \mu \mathrm{m}$. The diamonds represent 2MASS $J, H$, and $K_{\mathrm{s}}$-band fluxes and ISO synthetic photometry for $W 1, W 2, W 3$, and $W 4$. The dotted lines are the best-fit models described in the text. The molecular features discussed in the text are marked.

source photometry limits for the WISE 4 bands are $2,1.5,-3$, and -4 mag. When they are brighter than this limit, all the pixels are saturatured and no unsaturated pixels are available to fit to the PSF. Therefore the PSF-fit photometry is quite unreliable for sources brighter than the photometry limit. Because AGB stars typically are bright IR objects, most of our AGB samples are brighter than the saturation limits, and therefore only PSF-fit photometry is available. This comparison suggests that the PSF-fit photometry can be directly calibrated to the ISO synthetic photometry in the $W 3$ and $W 4$ bands and becomes quite unreliable for almost all the considered bright ISO AGB stars in $W 1$ and $W 2$ bands. Our ISO samples are confined to a relatively bright synthetic $W 3$ magnitude range of $W 3<0.5$. To facilitate our calibration down to the WISE saturation limit of $3.8 \mathrm{mag}$, we assumed that the good correlation between the PSF-fit and ISO synthetic $W 3$ photometry can be extrapolated. To ensure a reliable extrapolation, we adopted the fit with unity slope (Eq. (2)). To compensate for the loss of the $W 1$ and $W 2$ band data for bright objects like our AGB stars, we developed a calibration strategy based on the use of the DUSTY model template introduced in Sect. 2.2.

To find the best-fit model to each source, we adopted the following fitting procedure: the goodness of fit was measured by the overall offset between observed and model magnitudes. For each object, each set of model magnitudes was shifted together to obtain the smallest offset from the observed magnitudes. The overall shift is then the average of the differences in the five bands. We assumed that an overall shift smaller than 0.4 mag consistutes a good fit. For comparison, the average root mean square observational uncertainties at these five bands are 0.165 and 0.198 mag for O-rich and C-rich AGB, respectively.

We obtained the $W 3$ and $W 4$ magnitudes of the ISO AGB sources by convolving ISO spectra with proper filterresponse curves. Figure 2 shows the ISO spectra as a solid line and the associated 2MASS $J, H, K_{\mathrm{s}}$ fluxes (squares) for one AGB star of each type. The dotted lines are the fitted model SEDs. The models fit the observations well in the bands of interest. The best-fit model parameters and deviation from observations are displayed in each panel. We note that the best-fit model does not fit the dust resonance features (such as $9.7 \mu \mathrm{m}$ and $18 \mu \mathrm{m}$ silicate features) very well, since we only have two synthetic photometry points to sample this part of the SED. 

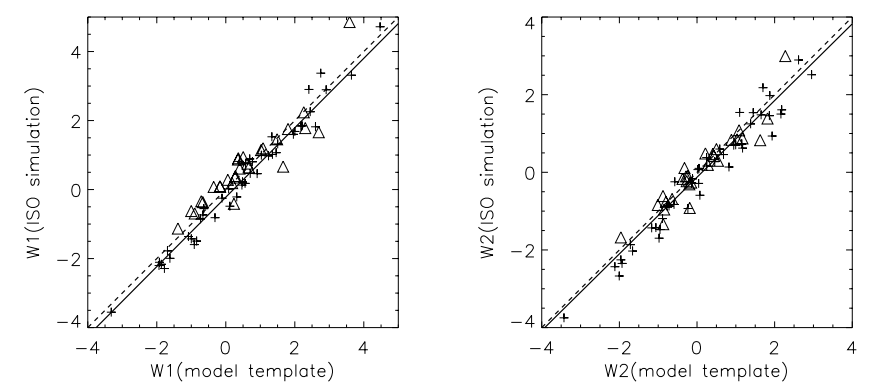

Fig. 3. Comparison between the ISO simulation magnitudes and the simulated magnitudes from the model for AGB stars in the WISE $W 1$ and $W 2$ bands. Pluses are O-rich and triangles are C-rich AGB stars. Dashed lines indicate equality between the $x$ - and $y$-axis values.

There are some other wavelength regions where model spectra do not match the observations, such as the absorption feature at about $5 \mu \mathrm{m}$ in the spectrum of the selected C-rich AGB star, which is absent from the model SED. The absorption feature at about $5 \mu \mathrm{m}$ may be the molecular features of $\mathrm{C}_{3}$ at $5.1 \mu \mathrm{m}$ and/or CO at $4.6 \mu \mathrm{m}$ (Gautschy-Loidl et al. 2004). The carbonstar spectrum shown in Fig. 2 also has deep features at $\sim 3 \mu \mathrm{m}$ and $\sim 14 \mu \mathrm{m}$, which are caused by $\mathrm{HCN}+\mathrm{C}_{2} \mathrm{H}_{2}$ (Gautschy-Loidl et al. 2004). Strong $\mathrm{C}_{2} \mathrm{H}_{2}$ features have been observed in carbon stars in nearby galaxies; in the Milky Way's high metallicity it is expected that there is a larger contribution from HCN to these features (Matsuura et al. 2006). More complicated DUSTY models with additional dust and molecular species may improve our fits. However, this requires significant efforts on choosing dust compositions for models and beyond the scope of the current work. The difference of the $W 2$ magnitude of this C-rich AGB star between observation and model is 0.11 mag. According to our analysis, this level of uncertainty does not significantly affect our results and our conclusions are still valid. We expect that the model spectra and dust properties can be better constrained with more photometry points in some narrowband filters that trace the molecular and dust features.

In total, 45 O-rich AGB and 28 C-rich AGB stars with ISO spectra were successfully modeled. The comparison of $W 1$ and $W 2$ magnitudes between ISO observations and the DUSTY models are shown in Fig. 3. Dashed lines indicate equality between the $x$ - and $y$-axis values. The data points are fitted with a linear equation as

$$
\begin{aligned}
& W 1(\text { ISO })=-0.21( \pm 0.07)+1.09( \pm 0.05) \times W 1(\text { model }) \\
& W 2(\text { ISO })=-0.15( \pm 0.06)+1.08( \pm 0.05) \times W 2(\text { model })
\end{aligned}
$$

The correlations of both O-rich and C-rich AGB stars (represented by pluses and triangles, respectively) are very close to the one-to-one equation, which suggests that the DUSTY models agree well with the ISO observations despite the degeneracy of some model parameters and no correction for interstellar extinction. The slight deviation from ISO synthetic photomety may be caused by the molecular features, which are absent in the model spectra.

Based on the above results, we calibrated our WISE AGB sample in five steps. First, we calibrated WISE $W 3$ and W4 PSF-fit photometry to ISO synthetic photometry by using Eq. (2). Second, we fit the calibrated $W 3$ and $W 4$ and the 2 MASS $J, H$, and $K_{\mathrm{s}}$-band magnitudes and determined the best DUSTY model for each WISE AGB star. Third, we compared the model $W 1$ and $W 2$ magnitudes with the observed ones for our whole sample of AGB stars and identified the magnitude ranges in which good linear correlations hold. Fourth, we assumed that the
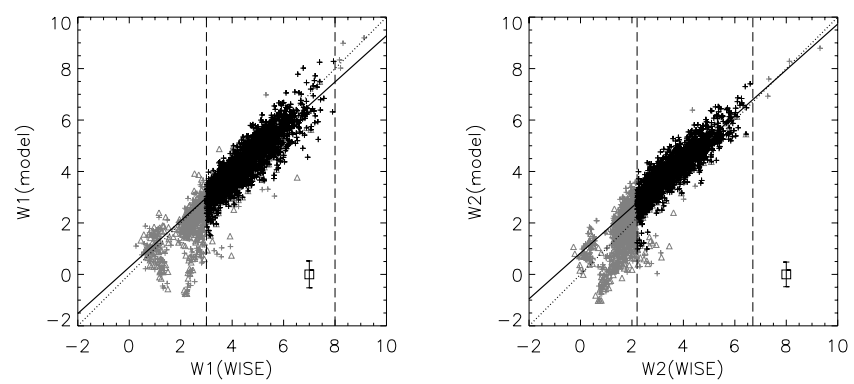

Fig. 4. Comparison between $W 1$ and $W 2$ magnitudes from the bestfit models and WISE observations. Vertical dashed lines delineate the magnitude limits between which AGB stars are selected to derive the coefficients of calibration equations. Solid lines are the linear leastsquares fits to the data points between lower limit and the WISE saturation limit. Dotted lines are the lines of the unit slope for reference. The error bars in the right corner indicate the median uncertainties in model magnitudes, including that caused by variability.

ISO simulated $W 1$ and $W 2$ magnitudes are reliable as a whole to derive empirical formulas for calibrating the observed $W 1$ and W2 magnitudes to those of the DUSTY model and then to those simulated by ISO. Finally, we applied the obtained calibration formulas to the observed $W 1$ and $W 2$ magnitudes of the objects in the good-correlation magnitude ranges. As a result, 1659 of 2203 O-rich and 835 of 958 C-rich WISE AGB stars meet our good-fit criteria. In total, we calibrated the $W 1 / W 2$ magnitudes of 2390/2021 AGB stars. The detailed calibration solution is discussed in Sect. 3.1. We note that about one fourth (544) of the O-rich AGB stars cannot be fitted by our model grid. We explored the model grid from Sargent et al. (2011) and found that the situation is not improved. Most of the objects without best-fits tend to have higher $W 3-W 4$ colors than fitted ones. Some even have an extreme $W 3$ - W4 color with strong deviations from the main distribution of O-rich AGB stars in the color-color diagram and seem to be misclassified as AGB stars or have some extreme properties that we do not know yet. We explored many possible model parameter ranges and did not find a significant improvement.

\section{Results}

\subsection{WISE calibration solution}

We show the comparison between the $W 1$ and $W 2$ model magnitudes and the WISE observation magnitudes in Fig. 4. A linear relationship seems to exist between the model values and the observations for most sources at the faint end, although these sources are all brighter than the nominal saturation limits. Only a small fraction of the sources at the bright end do not follow the relationships. The features at the bright end are similar to those in Fig. 1 when comparing ISO simulation magnitudes with WISE. This also supports the consistency between ISO spectra and DUSTY models. The faint end vertical dashed lines indicate the WISE saturation limits (8 and $6.7 \mathrm{mag}$ for $W 1$ and $W 2$ ). Vertical dashed lines at the bright end ( 3 and 2.2 mag for $W 1$ and $W 2$, respectively) indicate the criteria that are visually determined to exclude sources that disagree in the linear relationship. The bright useful limit is consistent with the WISE photometry limit mentioned in Sect. 2.3. Twenty data points in the $W 1$ and 32 data points in the $W 2$ panels, which significantly deviate from the linear distribution, were excluded. The correlation coefficients of the data points selected by the criteria and WISE saturation limit are 0.898 and 0.910 in the $W 1$ and $W 2$ bands. 
To acquire a more realistic relationship between model $W 1$ and $W 2$ and WISE $W 1$ and $W 2$, the uncertainties in the $y$-axis of the model synthetic magnitudes that are caused by variability of AGB stars need to be specified. We estimated the uncertainties caused by variability with the following three steps: first, we calculated a "typical" model SED with typical model parameters $\left(T_{\text {eff }}=3000 \mathrm{~K}, X=1, T_{\text {in }}=1000 \mathrm{~K}\right.$ and $\left.\tau_{0.55}=10\right)$. Then, for each band of $J, H, K_{\mathrm{s}}, W 3$, and $W 4$ of this "typical" model SED, we assumed a sinusoidal light curve with different amplitude and different phase. Smith et al. (2002) suggested that the variation amplitude decreases with increasing wavelength. Since the variability amplitude of the semiregular variables (SRVs) is typically $0.4-0.8 \mathrm{mag}$ and that of miras in the OGLE-III (Opitcal Graviational Lensing Experiment; Soszyński et al. 2013) dataset are higher than $1 \mathrm{mag}$ in the $I$ band, we assumed the typical amplitude of variation as $0.7,0.7,0.7,0.4$, and 0.4 mag for $J, H, K_{\mathrm{s}}$, $W 3$, and $W 4$ bands, respectively. We sampled each light curve at a random phase to generate a new SED. Finally, we simulated the new SED and compared the new $W 1$ and $W 2$ with the primary $W 1$ and $W 2$ of the "typical" model SED. We repeated the second and third steps to find the $W 1$ and $W 2$ model uncertainties, which are 0.522 and 0.480 mag.

To determine the linear relation in a more robust way, we used median fitting. The data points were divided into several bins with 150 objects in each bin along the $x$-axis. For each bin we computed the median $x$ - and $y$-values and used the median absolute deviation from the median (MADM) to estimate the spread in $x$ - and $y$-values. Then we fit a line through these new binned data points. The results of the linear equation using median fitting are as follows:

$$
\begin{aligned}
W 1(\text { model })= & 0.28( \pm 0.83) \\
& +0.90( \pm 0.17) \times W 1(\mathrm{WISE}) \\
W 2(\text { model })= & 0.83( \pm 0.67) \\
& +0.89( \pm 0.18) \times W 2(\mathrm{WISE}) .
\end{aligned}
$$

The linear relations are plotted as solid lines in the Fig. 4. The dotted lines of the unit slope are plotted for reference. The nonunity slope of linear relationships indicate that the offsets of the WISE observations are magnitude-dependent. Although several data points near the bright criteria do not follow the linear relationship, the fitted linear relations are valid for most data points. The dispersion may be caused by the variability of AGB stars and uncertainties in models. The relation between DUSTY models and ISO observations as described in Eq. (3) yields our final calibration formulas:

$$
\begin{aligned}
W 1(\text { calibrated })= & 0.10( \pm 0.91) \\
& +0.98( \pm 0.19) \times W 1(\text { WISE }) \\
W 2(\text { calibrated })= & 0.75( \pm 0.73) \\
& +0.96( \pm 0.20) \times W 2(\mathrm{WISE}) .
\end{aligned}
$$

The empirical calibration formulas are valid only in the ranges of 3-8 mag for $W 1$ and 2.2-6.7 mag for $W 2$. In our AGB star sample, the $W 1$ and $W 2$ magnitudes of 2390 and 2021 AGB stars are located in these ranges. The median of W(model)-W(PSF) is -0.024 and $0.557 \mathrm{mag}$ for $W 1$ and $W 2$. It can also be deduced from Eq. (5) that WISE PSF-fit photometry in general slightly underestimates the $W 1$ flux and overestimates the $W 2$ flux.

Several effects may contribute to the magnitude-dependent offsets. First, AGB stars have extended profiles because of their dusty mass-loss winds. The extended profile probably has an exaggerated effect on the PSF magnitudes that are computed using the unsaturated outer part of a saturated star image. Since brighter stars are typically more extended, this effect might contribute to the nonunity slope. Second, variability of AGB stars will introduce uncertainties in model simulation and dispersion in Fig. 4, which will reduce the slope. Finally, the flux-dependent shape of the PSF of the WISE detector in the saturated regime might be another contributor to the bright star flux biases, which are discussed in the section on photometric bias in the WISE Explanatory Supplement documentation of the All-Sky Data Release.

Since the uncertainties caused by variability of the $y$-axis are much larger than the uncertainties of observations in the $x$-axis, variability has a significant influence on the relation in Fig. 4. As we mentioned in Sect. 2.3, the molecular absorbtion feature in the $W 1$ and $W 2$ bands may be an important reason for the DUSTY model deviation from ISO, some molecules such as CO also have features in the near-infrared band. It is hard to quantify the effect of the molecular feature on the model simulation. However, changes caused by the molecular feature $(\sim 0.1 \mathrm{mag}$ in the $W 1$ and $W 2$ bands) to the infrared SED are much smaller than the change caused by variability $(\sim 0.5 \mathrm{mag}$ in the $W 1$ and $W 2$ bands). Since we have taken variability uncertainties into consideration, adding uncertainties caused by molecular features will not make a noticeable difference to the current results.

\subsection{Separation between $C$-rich and O-rich AGB}

We plot the $W 1-W 2$ versus $W 3-W 4$ color-color diagram of our calibrated WISE sample in Fig. 5. It can be seen that the two types of AGB stars are located in different regions in the figure and can be well distinguished in the WISE color-color diagram. A red solid line is plotted to effectively separate the two types of AGB stars. The straight lines successfully separate $87.1 \%$ of the O-rich AGB stars and $85.7 \%$ of the C-rich AGB stars. We computed the separation line by varying the slope and intercept until the product of the two separation fractions were minimum. This line function is

$W 1-W 2=2.35 \times(W 3-W 4)-1.24$.

It can be seen from Figure 5 that some data points lie far from the main distribution of AGB stars and show extremely high W3$W 4$ colors. They are the sample of objects whose SEDs cannot be successfully fit by our DUSTY model grids.

To probe the physical origin of this division, we calculated two sequences of DUSTY models by varying the optical depth while keeping the other three parameters fixed $\left(T_{\text {eff }}=3000 \mathrm{~K}\right.$, $X=1$ and $T_{\text {in }}=1000 \mathrm{~K}$ ) for both O-rich and C-rich AGB stars. The optical depth $\tau_{0.55}$ ranges from 0.01 to 100 . The resulting tracks are also plotted as green curves in the left panel of Fig. 5. In general, increasing the optical depth, which also indicates a decrease in the dust temperature, causes the data point to move diagonally from left bottom to upper right in the figure. O-rich models differ significantly from C-rich models in that the $W 1-W 2$ color increases much more slowly than $W 3-W 4$ does with increasing optical depth. Therefore, the distribution of O-rich models moves almost horizontally at the low $W 1-W 2$ end. The only difference between these two sequences of models is the dust composition. We also plot the blackbody curve for temperatures from $500 \mathrm{~K}$ to $3500 \mathrm{~K}$ as the dash-dotted line in the figure. The distribution of carbon stars roughly follows the trend of the blackbody curve, but with slightly bluer $W 1-W 2$ or redder $W 3-W 4$ colors than the latter.

The middle and right panels of Fig. 5 show the model SEDs with optical depth equal to $0.01,1.25$, and 40 for O-rich and C-rich AGB stars (middle panel for O-rich and right for 

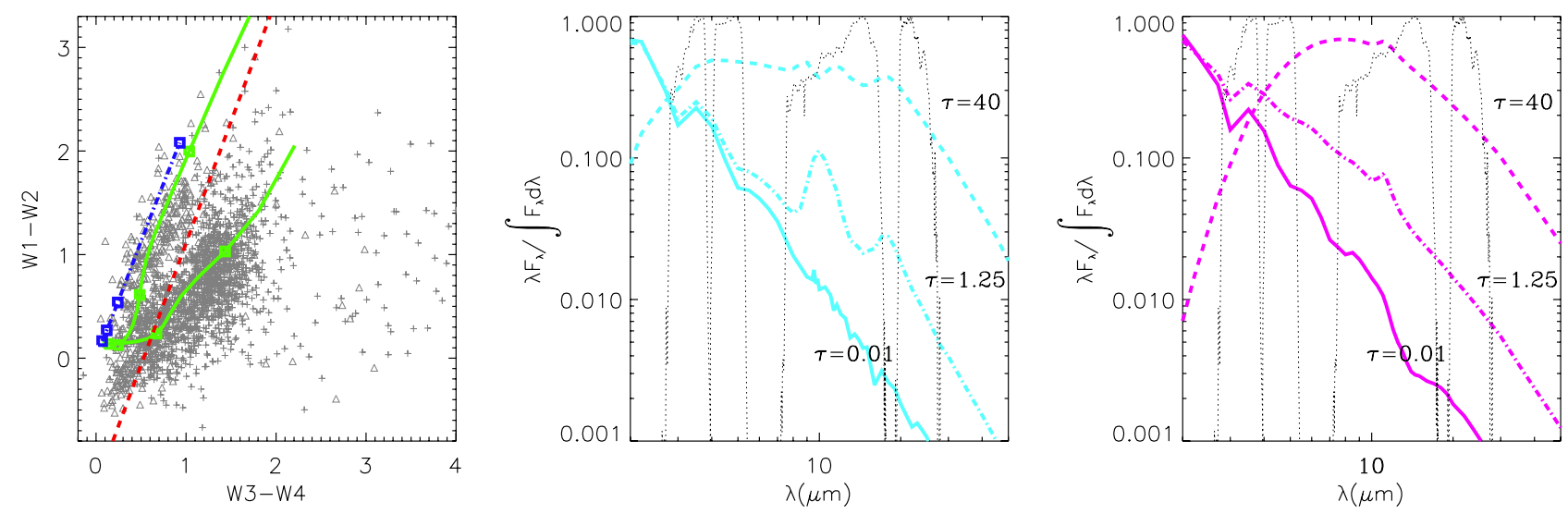

Fig. 5. Left panel: WISE color-color diagram that can be used to distinguish O-rich and C-rich AGB stars. Both the $W 1-W 2$ color and $W 3-$ W4 color are computed from the calibrated photometry. Pluses are O-rich stars, while triangles are C-rich stars. The dashed line is calculated to most effectively separate the two types of AGB stars. The solid green curves (left: O-rich, right: C-rich) are a series of models with different optical depths and typical values for the other 3 parameter values. The dash-dotted line is a series of blackbodies for reference. The four blue squares from bottom to top are blackbodies with temperatures of 3500, 2500, 1500, and $500 \mathrm{~K}$, respectively. The middle and right panels show the DUSTY model SEDs with optical depth $\tau_{0.55}=0.01,1.25$, and 80 for O-rich AGB and C-rich AGB, respectively. The dotted curves are the WISE $W 1-W 4$ band relative spectral response functions. The simulated colors of these four models are designated as thick squares in the left panel.

C-rich) and WISE $W 1-W 4$ response functions. The simulated WISE colors of these four models are indicated as squares in the left panel of Fig. 5. The SED of the C-rich star model with an optical depth of 0.01 almost overlaps that of the O-rich model with the same optical depth, since both of them are not affected significantly by dust and are essentially the spectrum of the central star. In contrast, SEDs of the models with an optical depth of 1.25 and 40 are different between the two AGB types, although the SEDs are normalized to the bolometric luminosities of the stars. When $\tau=1.25$, the O-rich star model shows negligible emission at the shorter wavelengths and thus is still dominated by the central star emission, while the C-rich star model has begun to be dominated by dust emission at these wavelengths. Conversely, O-rich AGB stars show stronger dust emission at longer wavelengths than C-rich AGB stars. This difference arises because O-rich dust species have much less efficient absorption at shorter wavelengths but more efficient absorption at longer wavelengths than C-rich dust species. This explains that the $W 1-W 2$ colors of O-rich model AGB stars are not sensitive to the increase of optical depth in the not very optically thick cases while their $W 3-W 4$ colors redden faster than C-rich AGB stars. When the circumstellar envelope becomes very optically thick (with $\tau=40$ ), the O-rich AGB star model still has bluer $W 1-W 2$ and redder $W 3-W 4$ colors than the C-rich AGB star model. This is because of the more efficient absorption at longer wavelengths and perhaps also because of the selfabsorption feature at $9.8 \mu \mathrm{m}$. The C-rich AGB stars are located closer to the blackbody line because the $\mathrm{C}$-rich dust species have a relatively smooth opacity profile.

\subsection{Model parameters}

From Fig. 5, it can be seen that the trend of our WISE AGB sample from left bottom to upper right in the color-color diagram is predominantly caused by changes in optical depth. To analyze the effects of the other three model parameters $\left(T_{\mathrm{eff}}, T_{\text {in }}\right.$ and $X$ ) on the mid-infrared colors, we took the optical depth tracks in Fig. 5 as standard model tracks and constructed new tracks for various values of the other three parameters. The three panels of Fig. 6 compare the model tracks produced by varying one of the three other model parameters. Note that not all values of $X$ might be physical. The maximum fractional abundance of $\mathrm{SiC}$ in the dust depends on the metallicity as well as on the fraction of Si that condenses into dust. The AmC-to-SiC ratio is also controlled by the dust condensation sequence; depending on whether $\mathrm{C}$ or $\mathrm{SiC}$ condenses first (see, e.g., Sect. 5 in Leisenring et al. (2008) and references therein). These model curves are only used to show the range of variation in our models that is caused by changes in the model parameters. Solid lines are the standard model tracks as shown in Fig. 5.

In the left panel of Fig. 6, it can be seen that the change of $T_{\text {eff }}$ mainly affects the optically thin objects. This is because the NIR SEDs of optically thin AGB stars are dominated by the central star's radiation. In the middle panel of Fig. 6, the change of the dust composition ratio $X$ mainly affects the IR colors of C-rich AGB star models while its effects are quite weak on O-rich AGB star models. This may be because the difference between the extinction curve of the two assumed dust components is steeper in C-rich AGB stars than that in O-rich AGB stars. In the right panel of Fig. 6, models with higher $T_{\text {in }}$ have fainter (i.e. bluer) $W 3-W 4$ and $W 1-W 2$ colors, which is consistent with the behavior of the blackbody. The variety of these physical parameters in AGB circumstellar envelopes contributes significantly to the data scatter in the WISE color-color diagram.

\subsection{Implications of the number statistics}

Because this is compilation of different works, it is hard to quantitatively estimate the completeness of our WISE AGB sample. However, we can determine the completeness from the spatial distribution of the sources. Most of the WISE AGB stars are located at the low Galactic latitude region, as expected. The longitude distributions are shown in Fig. 7. The left panel shows the whole sample, while the right two panels show sources with optical depth $\tau_{0.55}>10$ and $\tau_{0.55}<10$, respectively. It is obvious that more O-rich AGB stars (solid) concentrate toward the direction of the Galactic center, while C-rich AGB stars (dashed) are distributed more evenly along the Galactic longitude. The different longitude distributions of O-rich and C-rich AGB stars are consistent with previous works. For example, Le Bertre et al. (2003) found that C-rich AGB stars are located preferentially in the exterior of our Galaxy and O-rich stars tend to reside in 

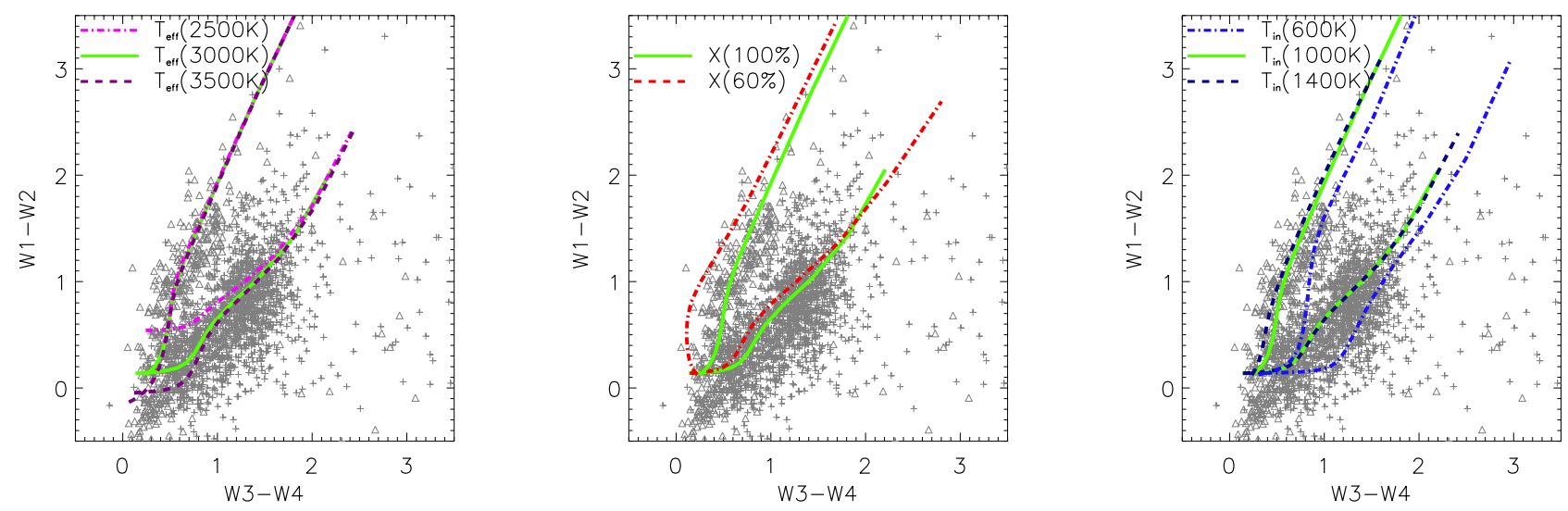

Fig. 6. WISE color-color diagram overlaid with models with different parameters. Models with the three parameters $T_{\text {eff }}, X$, and $T_{\text {in }}$ are shown in different panels from left to right. Solid lines are the fiducial model series as shown in Fig. 5. In each panel, the left set of tracks are models for C-rich stars and the right tracks plot O-rich models.
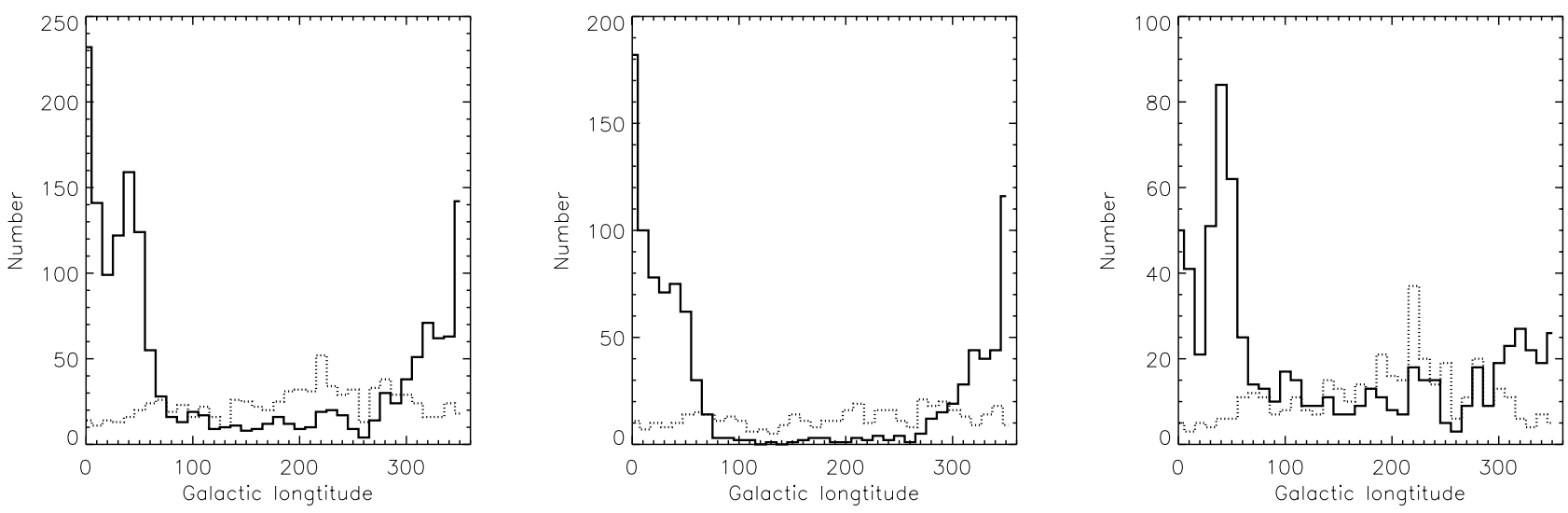

Fig. 7. Longtitude distribution of our calibrated WISE AGB stars. The left panel shows all stars in the sample, while the middle panel shows objects with $\tau_{0.55}>10$ and the right panel shows stars with $\tau_{0.55}<10$. O-rich AGB stars are solid lines and C-rich stars are plotted as dashed lines.

the interior of our Galaxy. The Galactic segregation of these two types of AGB stars is usually interpreted as an effect of the metallicity during the formation of C-rich AGB stars. Stellar evolution models of AGB stars show that O-rich AGB stars can evolve into $\mathrm{C}$-rich AGB stars more rapidly at lower metallicity (Le Bertre et al. 2003). Thus the metallicity gradient in our Galaxy can cause the different longtitude distributions of O-rich and C-rich AGB stars. The consistent spatial distributions between the our sample and other works suggests that the sample might represent a population of AGB stars in the solar neighborhood. This may be because the catalog is built from sources selected randomly in different works.

The middle panel of Fig. 7 is the longitude distribution of AGB stars with $\tau_{0.55}>10$. Very few high optical depth O-rich AGB stars locate in the direction opposed to the Galactic center. This deficiency of high optical depth object is obvious from comparing the middle panel and the right panel of Fig. 7. We can rule out the possibility that the differing Galactic interstellar extinction along different lines of sight could lead to this trend because no significant difference is present in the longitude distribution of low and high optical depth C-rich AGB stars. The metallcity gradient in our Galaxy may again be a natural explanation because O-rich AGB stars in the exterior of our Galaxy have lower metallicty and will evolve into C-rich AGB stars at earlier evolution times. These O-rich AGB stars may have a relatively lower amount of dust in the circumstellar envelopes and lower optical depth.

\section{Summary}

We reported our findings on AGB star colors in the WISE bands. We found that the WISE $W 1$ and $W 2$ magnitudes of AGB stars do not agree with the spectroscopic measurements from ISO when we compared our sample of ISO simulated $W 1-W 4$ magnitudes with WISE observations, which we attribute to the residual bias in the PSF-fit photometry of saturated objects in these two bands. The WISE saturated $W 3$ and $W 4$ magnitudes are directly calibrated based on ISO synthetic photometry. To calibrate the WISE $W 1$ and $W 2$ bands, we resorted to ISO spectra of a subsample of our AGB stars and proved that the radiation transfer code DUSTY can be used to reproduce unbiased $W 1$ and W2 magnitudes of bright stars. Using DUSTY, we successfully developed a calibration method for the observed WISE $W 1$ and $W 2$ bands to lift the residual bias of the PSF-fit photometry. The calibration procedure revealed that WISE may in general have underestimated $W 1$ flux and overestimated $W 2$ flux, and these deviations seem to be magnitude-dependent.

Combining the model-calibrated $W 1$ and $W 2$ data with the directly calibrated WISE $W 3$ and $W 4$ magnitudes, we analyzed the $W 1-W 2$ vs. $W 3-W 4$ color-color diagram and found that the two main types of AGB stars, O-rich AGB and C-rich AGB, can be effectively distinguished by their WISE colors. The division is mainly caused by the different extinction efficiencies between silicate-type and carbonaceous grains. The spatial distribution of the AGB sample is consistent with previous work. We also found 
that O-rich AGB stars with an opaque circumstellar envelope are much rarer toward the anti-Galactic center direction than C-rich AGB stars, which we attribute to the metallicity gradient of our Galaxy.

Acknowledgements. We thank the referee for thoughtful comments and insightful suggestions, which significantly improved the quality of this paper. This publication makes use of data products from the Wide-field Infrared Survey Explorer, which is a joint project of the University of California, Los Angeles, and the Jet Propulsion Laboratory/California Institute of Technology, funded by the National Aeronautics and Space Administration, and the Two Micron All Sky Survey, which is a joint project of the University of Massachusetts and the Infrared Processing and Analysis Center/California Institute of Technology, funded by the National Aeronautics and Space Administration and the National Science Foundation. We are grateful to Martin Cohen, who provided the Vega spectrum to check our magnitude simulation process, and to Edward Wright, who gave us very useful advice about the origin of the magnitude-dependent offset of WISE photometry in the saturated regime. This work is supported by China Ministry of Science and Technology under State Key Development Program for Basic Research (2012CB821800), the National Natural Science Foundation of China (NSFC, Nos. 11173056, 11225315 and 11320101002), Chinese Universities Scientific Fund (CUSF) and Specialized Research Fund for the Doctoral Program of Higher Education (SRFDP, No. 20123402110037).

\section{References}

Aringer, B., Girardi, L., Nowotny, W., Marigo, P., \& Lederer, M. T. 2009, A\&A, 503, 913

Begemann, B., Dorschner, J., Henning, T., et al. 1997, ApJ, 476, 199

Bloecker, T. 1995, A\&A, 297, 727

Boyer, M. L., Srinivasan, S., Riebel, D., et al. 2012, ApJ, 748, 40

Chu, Y. T., Bates, J. B., White, C. W., \& Farlow, G. C. 1988, J. Appl. Phys., 64, 3727

Cohen, M., Wheaton, W. A., \& Megeath, S. T. 2003, AJ, 126, 1090

Egan, M. P., Price, S. D., Kraemer, et al. 2003, Air Force Research Laboratory Technical Report AFRL-VS-TR-2003-1589

Gautschy-Loidl, R., Höfner, S., Jørgensen, U. G., \& Hron, J. 2004, A\&A, 422, 289

Groenewegen, M. A. T. 2006, A\&A, 448, 181

Groenewegen, M. A. T. 2012, A\&A, 543, A36
Habing, H. J. 1996, A\&ARv, 7, 97

Ivezic, Z., \& Elitzur, M. 1997, MNRAS, 287, 799

Jarrett, T. H., Cohen, M., Masci, F., et al. 2011, ApJ, 735, 112

Javadi, A., van Loon, J. T., Khosroshahi, H., \& Mirtorabi, M. T. 2013, MNRAS, 432, 2824

Jura, M., \& Kleinmann, S. G. 1989, ApJ, 341, 359

Kessler, M. F., Steinz, J. A., Anderegg, M. E., et al. 1996, A\&A, 315, L27

Koike, C., Yamamoto, T., Shibai, H., Kimura, S., \& Suto, H. 1995, Icarus, 114, 203

Kučinskas, A., Hauschildt, P. H., Ludwig, H.-G., et al. 2005, A\&A, 442, 281

Le Bertre, T., Tanaka, M., Yamamura, I., \& Murakami, H. 2003, A\&A, 403, 943

Leisenring, J. M., Kemper, F., \& Sloan, G. C. 2008, ApJ, 681, 1557

Lewis, B. M., Eder, J., \& Terzian, Y. 1990, ApJ, 362, 634

Maldoni, M. M., Ireland, T. R., Smith, R. G., \& Robinson, G. 2005, MNRAS, 362,872

Matsuura, M., Chesneau, O., Zijlstra, A. A., et al. 2006, ApJ, 646, L123

Mathis, J. S., Rumpl, W., \& Nordsieck, K. H. 1977, ApJ, 217, 425

Murakami, H., Bock, J., Freund, M. M., et al. 1994, ApJ, 428, 354

Neugebauer, G., Habing, H. J., van Duinen, R., et al. 1984, ApJ, 278, L1

Ossenkopf, V., Henning, T., \& Mathis, J. S. 1992, A\&A, 261, 567

Pegourie, B. 1988, A\&A, 194, 335

Posch, T., Mutschke, H., Trieloff, M., \& Henning, T. 2007, ApJ, 656, 615

Riebel, D., Srinivasan, S., Sargent, B., \& Meixner, M. 2012, ApJ, 753, 71

Riechers, D., Balega, Y., Driebe, T., et al. 2004, A\&A, 424, 165

Sargent, B. A., Srinivasan, S., \& Meixner, M. 2011, ApJ, 728, 93

Sloan, G. C., Kraemer, K. E., Price, S. D., \& Shipman, R. F. 2003, ApJS, 147, 379

Skrutskie, M. F., Cutri, R. M., Stiening, R., et al. 2006, AJ, 131, 1163

Smith, B. J., Leisawitz, D., Castelaz, M. W., \& Luttermoser, D. 2002, AJ, 123, 948

Soszyński, I., Udalski, A., Szymański, M. K., et al. 2013, Acta Astron., 63, 21

Speck, A. K., Corman, A. B., Wakeman, K., Wheeler, C. H., \& Thompson, G. 2009, ApJ, 691, 1202

Srinivasan, S., Sargent, B. A., \& Meixner, M. 2011, A\&A, 532, A54

Steffen, M., Szczerba, R., \& Schoenberner, D. 1998, A\&A, 337, 149

Suh, K.-W. 1999, MNRAS, 304, 389

Suh, K.-W. 2000, MNRAS, 315, 740

Suh, K.-W., \& Kim, H.-Y. 2002, A\&A, 391, 665

Suh, K.-W., \& Kwon, Y.-J. 2009, J. Korean Astron. Soc., 42, 81

Suh, K.-W., \& Kwon, Y.-J. 2011, MNRAS, 417, 3047 (Suh11)

Werner, M. W., Roellig, T. L., Low, F. J., et al. 2004, ApJS, 154, 1

Wright, E. L., Eisenhardt, P. R. M., Mainzer, A. K., et al. 2010, AJ, 140, 1868 